\title{
In Vitro Activity of Oral Antimicrobial Agents against Pathogens Associated with Community-Acquired Upper Respiratory Tract and Urinary Tract Infections: A Five Country Surveillance Study
}

\author{
Douglas J. Biedenbach • Robert E. Badal • Ming-Yi Huang • Mary Motyl • \\ Puneet K. Singhal · Roman S. Kozlov · Arthur Dessi Roman · Stephen Marcella
}

Received: April 12, 2016 / Published online: June 10, 2016

(C) The Author(s) 2016. This article is published with open access at Springerlink.com

\section{ABSTRACT}

Introduction: Bacterial infections that cause community-acquired urinary tract infections (CA-UTI) and upper respiratory tract infections (CA-URTI) are most frequently treated empirically. However, an increase in antimicrobial resistance has become a problem when treating outpatients.

Methods: This study determined the in vitro activities of oral antibiotics among 1501 pathogens from outpatients with CA-UTI and CA-URTI in medical centers during 2012 and

Enhanced content To view enhanced content for this article go to http://www.medengine.com/Redeem/ 44D4F0602B558BD5.

D. J. Biedenbach $(\bowtie) \cdot$ R. E. Badal

International Health Management Associates, Inc., Schaumburg, IL, USA

e-mail: dbiedenbach@ihmainc.com

M.-Y. Huang · M. Motyl · P. K. Singhal · S. Marcella Merck \& Co. Inc., Kenilworth, NJ, USA

R. S. Kozlov

Institute of Antimicrobial Chemotherapy, Smolensk

State Medical University, Smolensk, Russia

A. D. Roman

Research Institute for Tropical Medicine, Manila,

The Philippines
2013 from Argentina, Mexico, Venezuela, Russia, and the Philippines. Minimal inhibitory concentrations (MICs) were determined using broth microdilution and susceptibility defined by Clinical Laboratory Standards Institute (CLSI) and European Committee for Antimicrobial Susceptibility Testing (EUCAST) criteria.

Results: Ceftibuten $\left(\mathrm{MIC}_{50}, \leq 0.25 \mathrm{mg} / \mathrm{L}\right)$ was more potent in vitro compared to other $\beta$-lactams against Enterobacteriaceae from CA-UTI. Susceptibility to fluoroquinolones using CLSI criteria varied: Argentina and Mexico (50\%), the Philippines (60\%), Venezuela (70\%), and Russia (80\%). Fosfomycin susceptibility was $>90 \%$ against Enterobacteriaceae in each country. Susceptibility among Enterobacteriaceae to trimethoprim-sulfamethoxazole was 30.6-75.6\% and nitrofurantoin susceptibility also varied among the countries and was higher when EUCAST breakpoints were applied (65->90\%) compared to CLSI (52-84\%). All Haemophilus influenzae isolates from CA-URTI were susceptible to ceftibuten, cefixime, cefpodoxime, and cefuroxime using CLSI breakpoint criteria. EUCAST criteria produced intermediate and resistant MIC values for these oral 
cephalosporins. Country-specific susceptibility variation for fluoroquinolones, macrolides, and trimethoprim-sulfamethoxazole was observed among Streptococcus pneumoniae and Streptococcus pyogenes from CA-URTI.

Conclusion: This study demonstrated that antimicrobial susceptibility patterns varied in the five countries investigated among pathogens from CA-UTI and CA-URTI.

Funding: Merck \& Co. Inc., Kenilworth, New Jersey, USA.

Keywords: Community-acquired UTI and RTI; Oral antibiotics; Prescribing practices; Resistance

\section{INTRODUCTION}

Antimicrobial pressure, prescribing practices, and cultural factors, such as drug availability and cost, produce significant regional susceptibility differences among certain bacterial pathogens [1-6]. Individual countries first-line treatment options differ and may not be appropriate due to variable resistance patterns in local environments [2].

Country-specific surveillance data are available in most regions, but can be confounding based on the variation of results obtained from different investigations and differences in the susceptibility breakpoints applied in these studies. Regardless, surveillance studies provide useful information to primary care physicians who need to prescribe rational empiric therapy.

Community-acquired urinary tract infections (CA-UTI) and upper respiratory tract infections (CA-URTI) are the leading causes of outpatient infections and the most empirically treated infections worldwide $[1,6,7]$. Increased resistance to antibiotics has complicated the management of both of these outpatient infections. The spread of Enterobacteriaceae that carry a chromosomally mediated AmpC $\beta$-lactamase, an extended-spectrum $\beta$-lactamase (ESBL), or a carbapenemase is becoming a significant concern in the community [8-11]. Multidrug-resistant Streptococcus pneumoniae and $\beta$-hemolytic streptococci with tolerance to penicillin and resistance to macrolides and fluoroquinolones are increasing in Japan and extreme drug-resistant $S$. pneumoniae have been observed among multidrug-resistant isolates in Canada $[12,13]$.

Regional and country variations in pathogen resistance and the susceptibility breakpoint differences that are applied must be considered when determining empiric treatment options for both CA-UTI and CA-URTI [14, 15]. Many countries' adopt or design their own recommendations of treatment guidelines based on local surveillance data which can be scarce in some countries [1-6].

The objective of this study was to investigate the contemporary pattern of antimicrobial susceptibility among pathogens causing CA-UTI and CA-URTI in Argentina, Mexico, Venezuela, Russia, and the Philippines. These five countries have limited local surveillance data available for understanding susceptibility patterns among orally prescribed antimicrobial agents for common outpatient infections.

\section{METHODS}

Microbiology laboratories in five countries which have scare surveillance data were recruited to collect isolates from community-acquired infections. Only the first isolate collected from a patient who was in a 
clinic, a physician's office, a hospital emergency room, or in a community hospital for $<48 \mathrm{~h}$ at the time of collection was included. Patients were to have had no prior antibiotic exposure within the previous 90 days. Bacterial species from patients with CA-UTI included Escherichia coli, Klebsiella spp., Proteus mirabilis, and other less common Enterobacteriaceae. Species from CA-URTI included S. pneumoniae, Streptococcus pyogenes, $H$. influenzae, and $M$. catarrhalis, and a limited number of Enterobacteriaceae. A total of 1501 strains were collected during 2012 (29\%) and 2013 (71\%) from 12 medical centers, including two each in Argentina, Mexico, Venezuela, and the Philippines and four in Russia.

Identification of the bacterial isolates at the study site was performed using routine laboratory procedures and confirmed by matrix-assisted laser desorption ionization-time of flight (MALDI-TOF) mass spectrometry (MALDI Biotyper, Microflex, Bruker Daltonik GmbH, Bremen, Germany) at a reference laboratory (International Health Management Associates [IHMA], Schaumburg, IL, USA). Minimal inhibitory concentrations (MIC) were determined using the Clinical Laboratory Standards Institute (CLSI) broth microdilution procedure and panels prepared by IHMA using CLSI guidelines [15]. Susceptibility breakpoints used were those according to the CLSI and European Committee for Antimicrobial Susceptibility Testing (EUCAST) guidelines [16, 17]. Applied EUCAST breakpoints were those utilized for uncomplicated urinary tract infections which included amoxicillin-clavulanic acid, cefuroxime (oral dosing), cefixime, cefpodoxime, ceftibuten, fosfomycin (oral dosing), and nitrofurantoin. The oral CLSI cefuroxime interpretive criteria were used for Enterobacteriaceae. E. coli ATCC 25922,
Pseudomonas aeruginosa ATCC 27853, Staphylococcus aureus ATCC 29213, H. influenzae ATCC 49247, and S. pneumoniae ATCC 49619 were used as quality control (QC) strains. The QC MIC ranges utilized were those of the CLSI [18]. Isolates with an ESBL phenotype were confirmatory tested using cefotaxime \pm clavulanic acid and ceftazidime \pm clavulanic acid. The confirmatory test was performed on all E. coli, Klebsiella pneumoniae, Klebsiella oxytoca, and $P$. mirabilis with resistance to cefpodoxime (MICs $>1 \mathrm{mg} / \mathrm{L}$ ) [18].

This article does not contain any new studies with human or animal subjects performed by any of the authors.

\section{RESULTS}

Among the 960 isolates collected from all infection sources with a patient age provided, 331 were from pediatric patients and 629 were from adults ( $\geq 18$ years old). With respect to patient gender, among the 957 patients with this information recorded, 601 were female and 356 were male.

Enterobacteriaceae from CA-UTI included 345 isolates of E. coli, $87 \mathrm{~K}$. pneumoniae, $68 \mathrm{P}$. mirabilis, 27 Enterobacter cloacae, and 40 isolates of other species. Isolates were obtained from patients aged $0-17 \quad(n=76), \quad 18-39$ $(n=186), \quad 40-59 \quad(n=110), \quad 60-79$ years $(n=150)$, and $\geq 80$ years $(n=45)$. Further, approximately $75 \%$ of the isolates were obtained from women, and $23 \%$ of these were from women aged $\geq 65$ years. Among the 407 isolates collected from CA-URTI, $64.4 \%$ were collected from pediatric patient infections.

Ceftibuten $\left(\mathrm{MIC}_{50}, \leq 0.25 \mathrm{mg} / \mathrm{L}\right)$ was the most potent oral cephalosporin against Enterobacteriaceae from CA-UTI in each country 
(Table 1). The difference between the susceptibility rates of ceftibuten between countries ranged from $75.2 \%$ in the Philippines to $93.1 \%$ in Russia using CLSI criteria and from $71.7 \%$ (the Philippines) to 91.3\% (Russia) using EUCAST criteria. Cefuroxime, cefixime, cefpodoxime, and cefaclor were less active with a rank order of potency $\left(\mathrm{MIC}_{50}\right)$ as follows; cefixime $(0.5 \mathrm{mg} /$ L) $>$ cefpodoxime $\quad(0.5-1 \mathrm{mg} / \mathrm{L})>$ cefuroxime $(4-8 \mathrm{mg} / \mathrm{L})>$ cefaclor $\quad(4-16 \mathrm{mg} / \mathrm{L}) \quad$ and susceptibility rates lower than ceftibuten in all countries. Susceptibility to amoxicillin-clavulanic acid was $<60 \%$ in all countries with the exception of Russia (75.6\%). Susceptibility to norfloxacin and ciprofloxacin was similar and varied from approximately 50\% in Argentina and Mexico, $60 \%$ in the Philippines, $70 \%$ in Venezuela, and $80 \%$ in Russia. Trimethoprim-sulfamethoxazole susceptibility was highest in Russia (75.6\%) and lowest in Mexico (30.6\%). Susceptibility among E. coli to nitrofurantoin was high $(\geq 95 \%)$ using EUCAST breakpoints. However, susceptibility among combined Enterobacteriaceae species ranged from 51.5\% (Argentina) to $83.3 \%$ (Russia) using CLSI breakpoint criteria. All Enterobacteriaceae isolated from CA-URTI were susceptible to fluoroquinolones using CLSI but $<80 \%$ using EUCAST breakpoints while susceptibility percentages ranged from $64 \%$ to $79 \%$ for the other agents tested using either CLSI or EUCAST breakpoints (data on file, IHMA Inc.).

ESBL percentages for $E$. coli ranged from 9.2\% in Venezuela to $40.7 \%$ in Mexico. Similarly, ESBL percentages for $K$. pneumoniae were lowest $(18.8 \%)$ in Venezuela and highest (46.4\%) in Mexico. ESBL-producing $P$. mirabilis ranged from $10 \%$ to $33 \%$ with the highest percentage observed in Argentina. Fosfomycin and nitrofurantoin retained $>90 \%$ susceptibility against ESBL-positive E. coli in each country (data on file, IHMA Inc.). The activity of fosfomycin and nitrofurantoin diminished against ESBL-positive K. pneumoniae. Among the $\beta$-lactams tested, ceftibuten and amoxicillin-clavulanic acid provided the highest susceptibility percentages.

All $H$. influenzae isolates were susceptible to ceftibuten, cefixime, cefpodoxime, and cefuroxime using CLSI breakpoint criteria (Table 2). Intermediate and resistant MIC values were observed for each of these oral cephalosporins utilizing EUCAST breakpoint criteria. Reduced activity of amoxicillin/clavulanic acid was observed among $H$. influenzae isolates collected in Argentina and Mexico. One of three Haemophilus parainfluenzae from the Philippines had high-level resistant MIC values for all tested fluoroquinolones (data on file, IHMA Inc.). Isolates of $M$. catarrhalis were only collected in Argentina and all demonstrated a common broad spectrum susceptible pattern to the agents tested.

Streptococcus pneumoniae collected from Argentina, Mexico, and Russia were more susceptible to amoxicillin with and without clavulanic acid compared to other $\beta$-lactams (Table 2). Susceptibility oral cephalosporins were higher in Argentina compared to Russia and Mexico. Macrolide susceptibility was highest in Argentina. Levofloxacin susceptibility was high in all countries, though fluoroquinolone-resistant $S$. pneumoniae isolates were observed in all countries. Trimethoprim-sulfamethoxazole susceptibility was considerably lower in Russia (28-38\%) compared to Mexico (43-57\%) and Argentina (74-85\%) dependent upon the applied breakpoint criteria. All agents had activity against $S$. pyogenes. Azithromycin- and clarithromycin-resistant $S$. pyogenes was only 
Table 1 Susceptibility rates and MIC values for Enterobacteriaceae collected from CA-UTI

\begin{tabular}{|c|c|c|c|c|c|c|}
\hline \multirow{2}{*}{ Country $(n) /$ drug } & \multicolumn{3}{|l|}{ CLSI/EUCAST } & \multirow[t]{2}{*}{$\mathrm{MIC}_{50}$} & \multirow[t]{2}{*}{$\mathrm{MIC}_{90}$} & \multirow[t]{2}{*}{ MIC range } \\
\hline & $\% S$ & $\%$ I & $\% \mathrm{R}$ & & & \\
\hline \multicolumn{7}{|l|}{ Argentina (101) } \\
\hline Amoxicillin/clavulanic acid & $52.5 / 82.2$ & $14.9 /-$ & $32.7 / 17.8$ & 8 & $>32$ & $\leq 1->32$ \\
\hline Ceftibuten & $83.2 / 71.3$ & $3.0 /-$ & $13.9 / 28.7$ & 0.25 & $>16$ & $\leq 0.06->16$ \\
\hline Cefixime & $58.4 / 58.4$ & $6.9 /-$ & $34.7 / 41.6$ & 0.5 & $>8$ & $\leq 0.12->8$ \\
\hline Cefpodoxime & $56.4 / 53.5$ & $5.0 /-$ & $38.6 / 46.5$ & 1 & $>8$ & $\leq 0.12->8$ \\
\hline Cefuroxime & $43.6 / 50.5$ & $13.9 /-$ & $42.6 / 49.5$ & 8 & $>32$ & $\leq 1->32$ \\
\hline Cefaclor & $47.5 / \mathrm{NA}$ & $6.9 / \mathrm{NA}$ & $45.5 / \mathrm{NA}$ & 16 & $>32$ & $\leq 0.5->32$ \\
\hline Ciprofloxacin & $50.5 / 49.5$ & $49.5 / 1.0$ & $0.0 / 49.5$ & 1 & $>1$ & $\leq 0.002->1$ \\
\hline Norfloxacin & $50.5 / 38.6$ & $1.98 / 6.9$ & $47.5 / 54.5$ & 4 & $>8$ & $0.03->8$ \\
\hline Fosfomycin & $91.1 / 84.2$ & $1.0 /-$ & $7.9 / 15.8$ & 4 & 64 & $\leq 0.25->128$ \\
\hline Nitrofurantoin & $51.5 / 64.4(100)^{\mathrm{a}}$ & $12.9 /-$ & $35.6 / 35.6$ & 32 & $>128$ & $\leq 2->128$ \\
\hline Trimethoprim/sulfamethoxazole & $49.5 / 49.5$ & $0.0 / 1.0$ & $50.5 / 49.5$ & 4 & $>64$ & $\leq 0.5->64$ \\
\hline \multicolumn{7}{|l|}{ Mexico (98) } \\
\hline Amoxicillin/clavulanic acid & $58.2 / 92.9$ & $28.6 /-$ & $13.3 / 7.1$ & 8 & 32 & $\leq 1->32$ \\
\hline Ceftibuten & $77.6 / 66.3$ & $6.1 /-$ & $16.3 / 33.7$ & 0.25 & $>16$ & $\leq 0.06->16$ \\
\hline Cefixime & $58.2 / 58.2$ & $6.1 /-$ & $35.7 / 41.8$ & 0.5 & $>8$ & $\leq 0.12->8$ \\
\hline Cefpodoxime & $60.2 / 54.1$ & $3.1 /-$ & $36.7 / 45.9$ & 1 & $>8$ & $\leq 0.12->8$ \\
\hline Cefuroxime & $43.9 / 58.2$ & $16.3 /-$ & $39.8 / 41.8$ & 8 & $>32$ & $\leq 1->32$ \\
\hline Cefaclor & $45.9 / \mathrm{NA}$ & $7.1 / \mathrm{NA}$ & $46.9 / \mathrm{NA}$ & 16 & $>32$ & $\leq 0.5->32$ \\
\hline Ciprofloxacin & $51.0 / 45.9$ & $50.0 / 5.1$ & $0.0 / 49.0$ & 1 & $>1$ & $0.004->1$ \\
\hline Norfloxacin & $48.0 / 37.8$ & $6.1 / 4.1$ & $45.9 / 58.2$ & 8 & $>8$ & $0.03->8$ \\
\hline Fosfomycin & $95.9 / 90.8$ & $1.0 /-$ & $3.1 / 9.2$ & 2 & 32 & $\leq 0.25->128$ \\
\hline Nitrofurantoin & $65.3 / 80.6(94.7)^{c}$ & $15.3 /-$ & $19.4 / 19.4$ & 16 & $>128$ & $\leq 2->128$ \\
\hline Trimethoprim/sulfamethoxazole & $30.6 / 30.6$ & $0.0 / 3.1$ & $69.4 / 66.3$ & $>64$ & $>64$ & $\leq 0.5->64$ \\
\hline \multicolumn{7}{|l|}{ Venezuela (95) } \\
\hline Amoxicillin/clavulanic acid & $55.8 / 88.4$ & $26.3 /-$ & $17.9 / 11.6$ & 8 & $>32$ & $\leq 1->32$ \\
\hline Ceftibuten & $89.5 / 84.2$ & $2.1 /-$ & $8.4 / 15.8$ & 0.25 & 16 & $\leq 0.06->16$ \\
\hline Cefixime & $74.7 / 74.7$ & $8.4 /-$ & $16.8 / 25.3$ & 0.5 & $>8$ & $\leq 0.12->8$ \\
\hline Cefpodoxime & $82.1 / 76.8$ & $1.1 /-$ & $16.8 / 23.2$ & 0.5 & $>8$ & $\leq 0.12->8$ \\
\hline Cefuroxime & $52.6 / 77.9$ & $29.5 /-$ & $17.9 / 22.1$ & 4 & $>32$ & $\leq 1->32$ \\
\hline Cefaclor & 64.2/NA & 6.3/NA & $29.5 / \mathrm{NA}$ & 4 & $>32$ & $\leq 0.5->32$ \\
\hline
\end{tabular}


Table 1 continued

\begin{tabular}{|c|c|c|c|c|c|c|}
\hline \multirow[t]{2}{*}{ Country $(n) /$ drug } & \multicolumn{3}{|l|}{ CLSI/EUCAST } & \multirow[t]{2}{*}{$\mathrm{MIC}_{50}$} & \multirow[t]{2}{*}{$\mathrm{MIC}_{90}$} & \multirow[t]{2}{*}{ MIC range } \\
\hline & $\% S$ & $\%$ I & $\% \mathbf{R}$ & & & \\
\hline Ciprofloxacin & $72.6 / 72.6$ & $27.4 / 0.0$ & $0.0 / 27.4$ & 0.015 & $>1$ & $\leq 0.002->1$ \\
\hline Norfloxacin & $72.6 / 63.2$ & $0.0 / 4.2$ & $27.4 / 32.6$ & 0.12 & $>8$ & $\leq 0.015->8$ \\
\hline Fosfomycin & $92.6 / 88.4$ & $3.2 /-$ & $4.2 / 11.6$ & 2 & 64 & $\leq 0.25->128$ \\
\hline Nitrofurantoin & $68.4 / 81.1(97.0)^{\mathrm{c}}$ & $12.6 /-$ & $19.0 / 19.0$ & 16 & 128 & $4->128$ \\
\hline Trimethoprim/sulfamethoxazole & $57.9 / 57.9$ & $0.0 / 1.1$ & $42.1 / 41.1$ & $\leq 0.5$ & $>64$ & $\leq 0.5->64$ \\
\hline \multicolumn{7}{|l|}{ Russia (160) } \\
\hline Amoxicillin/clavulanic acid & $75.6 / 95.6$ & $15.6 /-$ & $8.8 / 4.4$ & 4 & 16 & $\leq 1->32$ \\
\hline Ceftibuten & $93.1 / 91.3$ & $0.6 /-$ & $6.3 / 8.7$ & 0.12 & 1 & $\leq 0.06->16$ \\
\hline Cefixime & $81.3 / 81.3$ & $3.1 /-$ & $15.6 / 18.7$ & 0.5 & 8 & $\leq 0.12->8$ \\
\hline Cefpodoxime & $81.9 / 80.0$ & $1.2 /-$ & $16.9 / 20.0$ & 0.5 & $>8$ & $\leq 0.12->8$ \\
\hline Cefuroxime & $63.7 / 79.4$ & $16.9 /-$ & $19.4 / 20.6$ & 4 & $>32$ & $\leq 1->32$ \\
\hline Cefaclor & 76.2/NA & $1.9 / \mathrm{NA}$ & 21.9/NA & 4 & $>32$ & $\leq 0.5->32$ \\
\hline Ciprofloxacin & $80.0 / 79.4$ & $20.0 / 0.6$ & $0.0 / 20.0$ & 0.015 & $>1$ & $0.004->1$ \\
\hline Norfloxacin & $81.9 / 76.9$ & $0.0 / 2.5$ & $18.1 / 20.6$ & 0.06 & $>8$ & $0.03->8$ \\
\hline Fosfomycin & $98.1 / 95.6$ & $0.6 /-$ & $1.3 / 4.4$ & 1 & 16 & $\leq 0.25->128$ \\
\hline Nitrofurantoin & $83.8 / 91.9(98.4)^{c}$ & $8.1 /-$ & $8.1 / 8.1$ & 16 & 64 & $\leq 2->128$ \\
\hline Trimethoprim/sulfamethoxazole & $75.6 / 75.6$ & $0.0 / 0.6$ & $24.4 / 23.8$ & $\leq 0.5$ & $>64$ & $\leq 0.5->64$ \\
\hline \multicolumn{7}{|l|}{ Philippines (113) } \\
\hline Amoxicillin/clavulanic acid & $54.0 / 84.1$ & $16.8 /-$ & $29.2 / 15.9$ & 8 & $>32$ & $\leq 1->32$ \\
\hline Ceftibuten & $75.2 / 71.7$ & $7.1 /-$ & $17.7 / 28.3$ & 0.12 & $>16$ & $\leq 0.06->16$ \\
\hline Cefixime & $62.8 / 62.8$ & $3.5 /-$ & $33.6 / 37.2$ & 0.5 & $>8$ & $\leq 0.12->8$ \\
\hline Cefpodoxime & $67.3 / 62.8$ & $2.7 /-$ & $30.1 / 37.2$ & 0.5 & $>8$ & $\leq 0.12->8$ \\
\hline Cefuroxime & $48.7 / 59.3$ & $16.8 /-$ & $34.5 / 40.7$ & 8 & $>32$ & $\leq 1->32$ \\
\hline Cefaclor & 54.0/NA & $3.5 / \mathrm{NA}$ & $42.5 / \mathrm{NA}$ & 4 & $>32$ & $\leq 0.5->32$ \\
\hline Ciprofloxacin & $62.0 / 59.3$ & $38.1 / 2.7$ & $0 / 38.1$ & 0.12 & $>1$ & $\leq 0.002->1$ \\
\hline Norfloxacin & $63.7 / 54.9$ & $8.9 / 1.8$ & $27.4 / 43.4$ & 0.5 & $>8$ & $\leq 0.015->8$ \\
\hline Fosfomycin & $91.2 / 83.2$ & $3.5 /-$ & $5.3 / 16.8$ & 4 & 64.0 & $\leq 0.25->128$ \\
\hline Nitrofurantoin & $53.1 / 73.5(96.3)^{c}$ & $20.4 /-$ & $26.6 / 26.6$ & 32 & 128.0 & $\leq 2->128$ \\
\hline
\end{tabular}


Table 1 continued

\begin{tabular}{|c|c|c|c|c|c|c|}
\hline \multirow[t]{2}{*}{ Country $(n) /$ drug } & \multicolumn{3}{|c|}{ CLSI/EUCAST } & \multirow[t]{2}{*}{$\mathrm{MIC}_{50}$} & \multirow[t]{2}{*}{$\mathrm{MIC}_{90}$} & \multirow[t]{2}{*}{ MIC range } \\
\hline & $\% S$ & $\%$ I & $\% \mathbf{R}$ & & & \\
\hline Trimethoprim/sulfamethoxazole & $44.3 / 44.3$ & $0 / 1.8$ & $55.8 / 54.0$ & $>64$ & $>64$ & $\leq 0.5->64$ \\
\hline
\end{tabular}

Number in parentheses is the susceptibility rates when applied to E. coli only (EUCAST recommendation)

CLSI Clinical Laboratory Standards Institute, EUCAST European Committee for Antimicrobial Susceptibility Testing, I intermediate, $M I C$ Minimal inhibitory concentrations, $R$ resistant, $S$ susceptible, $C A$-UTI community-acquired urinary tract infection, NA or a dash $=$ No interpretive breakpoints available

Table 2 Susceptibility and MIC values for fastidious respiratory tract pathogens from CA-URTI

\begin{tabular}{|c|c|c|c|c|c|c|}
\hline \multirow[t]{2}{*}{ Organism } & \multirow[t]{2}{*}{ Country $(n) /$ drug } & \multicolumn{3}{|c|}{ CLSI/EUCAST } & \multirow[t]{2}{*}{$\mathrm{MIC}_{50}$} & \multirow[t]{2}{*}{$\mathrm{MIC}_{90}$} \\
\hline & & $\% S$ & $\%$ I & $\% \mathrm{R}$ & & \\
\hline \multirow[t]{15}{*}{ S. pneumoniae } & Argentina (19) & & & & & \\
\hline & Amoxicillin & $100 / \mathrm{NA}$ & $0.0 / \mathrm{NA}$ & $0.0 / \mathrm{NA}$ & $\leq 0.12$ & 0.25 \\
\hline & Amoxicillin/clavulanic acid & $100 / \mathrm{NA}$ & $0.0 / \mathrm{NA}$ & $0.0 / \mathrm{NA}$ & $\leq 0.12$ & 0.25 \\
\hline & Ceftibuten & $\mathrm{NA} / \mathrm{NA}$ & $\mathrm{NA} / \mathrm{NA}$ & $\mathrm{NA} / \mathrm{NA}$ & $>4$ & $>4$ \\
\hline & Cefixime & $\mathrm{NA} / \mathrm{NA}$ & NA/NA & NA/NA & 0.25 & 2 \\
\hline & Cefpodoxime & $100 / 94.7$ & $0.0 / 5.3$ & $0.0 / 0.0$ & $\leq 0.03$ & 0.25 \\
\hline & Cefuroxime & $94.7 / 94.7$ & $5.3 / 0.0$ & $0.0 / 5.3$ & $\leq 0.03$ & 0.5 \\
\hline & Cefaclor & $84.2 / 0.0$ & $5.3 / 73.7$ & $10.5 / 26.3$ & 0.5 & 4 \\
\hline & Azithromycin & $78.9 / 0.0$ & $0.0 / 78.9$ & $21.1 / 21.1$ & $\leq 0.5$ & $>4$ \\
\hline & Clarithromycin & $78.9 / 78.9$ & $0.0 / 0.0$ & $21.1 / 21.1$ & $\leq 0.25$ & $>2$ \\
\hline & Ciprofloxacin & $\mathrm{NA} / 0.0$ & NA/100 & $\mathrm{NA} / 0.0$ & 0.5 & $>1$ \\
\hline & Levofloxacin & $94.7 / 94.7$ & $5.3 / 0.0$ & $0.0 / 5.3$ & 2 & 2 \\
\hline & Moxifloxacin & $100 / 100$ & $0.0 / 0.0$ & $0.0 / 0.0$ & 0.12 & 0.12 \\
\hline & Doxycycline & $84.2 / 94.7$ & $10.5 / 0.0$ & $5.3 / 5.3$ & $\leq 0.06$ & 0.5 \\
\hline & Trimethoprim/sulfamethoxazole & $73.7 / 84.2$ & $10.5 / 0.0$ & $15.8 / 15.8$ & 0.5 & $>2$ \\
\hline \multirow[t]{8}{*}{ S. pneumoniae } & $\operatorname{Mexico}(14)$ & & & & & \\
\hline & Amoxicillin & $71.4 / \mathrm{NA}$ & 7.1/NA & $21.4 / \mathrm{NA}$ & $\leq 0.12$ & $>16$ \\
\hline & Amoxicillin/clavulanic acid & $71.4 / \mathrm{NA}$ & $0.0 / \mathrm{NA}$ & $28.6 / \mathrm{NA}$ & $\leq 0.12$ & $>16$ \\
\hline & Ceftibuten & NA/NA & NA/NA & NA/NA & $>4$ & $>4$ \\
\hline & Cefixime & $\mathrm{NA} / \mathrm{NA}$ & NA/NA & NA/NA & 1 & $>8$ \\
\hline & Cefpodoxime & $64.3 / 57.1$ & $0.0 / 7.1$ & $35.7 / 35.7$ & 0.12 & $>4$ \\
\hline & Cefuroxime & $57.1 / 57.1$ & $14.3 / 0.0$ & $28.6 / 42.9$ & 0.25 & $>4$ \\
\hline & Cefaclor & $28.6 / 0.0$ & $28.6 / 21.4$ & $42.9 / 78.6$ & 2 & $>8$ \\
\hline
\end{tabular}


Table 2 continued

\begin{tabular}{|c|c|c|c|c|c|c|}
\hline \multirow[t]{2}{*}{ Organism } & \multirow[t]{2}{*}{ Country $(n) /$ drug } & \multicolumn{3}{|c|}{ CLSI/EUCAST } & \multirow[t]{2}{*}{$\mathrm{MIC}_{50}$} & \multirow[t]{2}{*}{$\mathrm{MIC}_{90}$} \\
\hline & & $\% S$ & $\%$ I & $\% \mathbf{R}$ & & \\
\hline & Azithromycin & $71.4 / 0.0$ & $0.0 / 71.4$ & $28.6 / 28.6$ & $\leq 0.5$ & $>4$ \\
\hline & Clarithromycin & $71.4 / 71.4$ & $0.0 / 0.0$ & $28.6 / 28.6$ & $\leq 0.25$ & $>2$ \\
\hline & Ciprofloxacin & $\mathrm{NA} / 0.0$ & NA/100 & NA/0.0 & 1 & $>1$ \\
\hline & Levofloxacin & $85.7 / 85.7$ & $0.0 / 0.0$ & $14.3 / 14.3$ & 2 & $>16$ \\
\hline & Moxifloxacin & $85.7 / 85.7$ & $0.0 / 0.0$ & $14.3 / 14.3$ & 0.12 & $>8$ \\
\hline & Doxycycline & $50.0 / 50.0$ & $0.0 / 0.0$ & $50 / 50.0$ & 0.12 & $>2$ \\
\hline & Trimethoprim/sulfamethoxazole & $42.9 / 57.1$ & $14.3 / 0.0$ & $42.9 / 42.9$ & 1 & $>2$ \\
\hline \multirow[t]{15}{*}{ S. pneumoniae } & Russia (148) & & & & & \\
\hline & Amoxicillin & 83.8/NA & $8.1 / \mathrm{NA}$ & $8.1 / \mathrm{NA}$ & $\leq 0.12$ & 4 \\
\hline & Amoxicillin/clavulanic acid & $83.8 / \mathrm{NA}$ & $6.1 / \mathrm{NA}$ & $10.1 / \mathrm{NA}$ & $\leq 0.12$ & $>4$ \\
\hline & Ceftibuten & NA/NA & NA/NA & NA/NA & $>4$ & $>4$ \\
\hline & Cefixime & NA/NA & NA/NA & NA/NA & 0.5 & $>8$ \\
\hline & Cefpodoxime & $69.6 / 67.6$ & $0.7 / 2.0$ & $29.7 / 30.4$ & 0.06 & $>4$ \\
\hline & Cefuroxime & $67.6 / 65.6$ & $1.3 / 2.0$ & $31.1 / 32.4$ & 0.12 & $>4$ \\
\hline & Cefaclor & $61.5 / 0.0$ & $3.4 / 50.7$ & $35.1 / 49.3$ & 0.5 & $>8$ \\
\hline & Azithromycin & $56.1 / 0.0$ & $0.7 / 56.1$ & $43.2 / 43.9$ & $\leq 0.5$ & $>4$ \\
\hline & Clarithromycin & $56.8 / 56.8$ & $0.0 / 0.0$ & $43.2 / 43.2$ & $\leq 0.25$ & $>2$ \\
\hline & Ciprofloxacin & NA/1.4 & NA/98.6 & $\mathrm{NA} / 0$ & 0.5 & 1 \\
\hline & Levofloxacin & $83.8 / 87.8$ & $16.2 / 0.0$ & $0.0 / 16.2$ & 2 & 4 \\
\hline & Moxifloxacin & $100 / 100$ & $0.0 / 0.0$ & $0.0 / 0.0$ & 0.12 & 0.12 \\
\hline & Doxycycline & $45.3 / 49.3$ & $2.7 / 11.5$ & $52.0 / 39.2$ & 2 & $>2$ \\
\hline & Trimethoprim/sulfamethoxazole & $28.4 / 38.5$ & $24.3 / 14.2$ & $47.3 / 47.3$ & 2 & $>2$ \\
\hline \multirow[t]{8}{*}{ S. pyogenes } & Argentina (20) & & & & & \\
\hline & Amoxicillin & NA/NA & NA/NA & NA/NA & $\leq 0.12$ & $\leq 0.12$ \\
\hline & Amoxicillin/clavulanic acid & NA/NA & NA/NA & NA/NA & $\leq 0.12$ & $\leq 0.12$ \\
\hline & Ceftibuten & NA/NA & NA/NA & $0.0 / \mathrm{NA}$ & 0.5 & 0.5 \\
\hline & Cefixime & NA/NA & NA/NA & NA/NA & $\leq 0.06$ & 0.12 \\
\hline & Cefpodoxime & NA/NA & NA/NA & NA/NA & $\leq 0.03$ & $\leq 0.03$ \\
\hline & Cefuroxime & NA/NA & NA/NA & NA/NA & $\leq 0.03$ & $\leq 0.03$ \\
\hline & Cefaclor & NA/NA & NA/NA & NA/NA & 0.12 & 0.12 \\
\hline
\end{tabular}


Table 2 continued

\begin{tabular}{|c|c|c|c|c|c|c|}
\hline \multirow[t]{2}{*}{ Organism } & \multirow[t]{2}{*}{ Country $(n) /$ drug } & \multicolumn{3}{|c|}{ CLSI/EUCAST } & \multirow[t]{2}{*}{$\mathrm{MIC}_{50}$} & \multirow[t]{2}{*}{$\mathrm{MIC}_{90}$} \\
\hline & & $\% S$ & $\%$ I & $\% \mathbf{R}$ & & \\
\hline & Azithromycin & $100 / 0.0$ & $0.0 / 100$ & $0.0 / 0.0$ & $\leq 0.5$ & $\leq 0.5$ \\
\hline & Clarithromycin & $100 / 100$ & $0.0 / 0.0$ & $0.0 / 0.0$ & $\leq 0.25$ & $\leq 0.25$ \\
\hline & Ciprofloxacin & NA/NA & $\mathrm{NA} / \mathrm{NA}$ & NA/NA & $\leq 0.12$ & 1 \\
\hline & Levofloxacin & $85.0 / 75.0$ & $15.0 / 10.0$ & $0.0 / 15.0$ & 1 & 4 \\
\hline & Moxifloxacin & NA/100 & NA/0.0 & $\mathrm{NA} / 0.0$ & $\leq 0.06$ & 0.25 \\
\hline & Doxycycline & NA/95.0 & $\mathrm{NA} / 0.0$ & $\mathrm{NA} / 5.0$ & $\leq 0.06$ & $\leq 0.06$ \\
\hline & Trimethoprim/sulfamethoxazole & NA/100 & NA/0.0 & NA/0.0 & $\leq 0.12$ & $\leq 0.12$ \\
\hline \multirow[t]{15}{*}{ S. pyogenes } & Mexico (20) & & & & & \\
\hline & Amoxicillin & NA/NA & NA/NA & NA/NA & $\leq 0.12$ & $\leq 0.12$ \\
\hline & Amoxicillin/clavulanic acid & NA/NA & NA/NA & NA/NA & $\leq 0.12$ & $\leq 0.12$ \\
\hline & Ceftibuten & NA/NA & NA/NA & NA/NA & 0.25 & 0.5 \\
\hline & Cefixime & NA/NA & NA/NA & NA/NA & $\leq 0.06$ & 0.12 \\
\hline & Cefpodoxime & NA/NA & NA/NA & NA/NA & $\leq 0.03$ & $\leq 0.03$ \\
\hline & Cefuroxime & NA/NA & NA/NA & NA/NA & $\leq 0.03$ & $\leq 0.03$ \\
\hline & Cefaclor & NA/NA & NA/NA & NA/NA & 0.12 & 0.12 \\
\hline & Azithromycin & $100 / 0.0$ & $0.0 / 100$ & $0.0 / 0.0$ & $\leq 0.5$ & $\leq 0.5$ \\
\hline & Clarithromycin & $100 / 100$ & $0.0 / 0.0$ & $0.0 / 0.0$ & $\leq 0.25$ & $\leq 0.25$ \\
\hline & Ciprofloxacin & NA/NA & $\mathrm{NA} / \mathrm{NA}$ & NA/NA & $\leq 0.12$ & 0.25 \\
\hline & Levofloxacin & $100 / 95.0$ & $0.0 / 5.0$ & $0.0 / 0.0$ & 1 & 1 \\
\hline & Moxifloxacin & NA/100 & $\mathrm{NA} / 0.0$ & $\mathrm{NA} / 0.0$ & $\leq 0.06$ & 0.12 \\
\hline & Doxycycline & NA/90.0 & $\mathrm{NA} / 0$ & $\mathrm{NA} / 10.0$ & $\leq 0.06$ & $\leq 0.06$ \\
\hline & Trimethoprim/sulfamethoxazole & NA/100 & $\mathrm{NA} / 0.0$ & $\mathrm{NA} / 0.0$ & $\leq 0.12$ & $\leq 0.12$ \\
\hline \multirow[t]{8}{*}{ S. pyogenes } & Philippines (11) & & & & & \\
\hline & Amoxicillin & NA/NA & NA/NA & NA/NA & $\leq 0.12$ & $\leq 0.12$ \\
\hline & Amoxicillin/clavulanic acid & $\mathrm{NA} / \mathrm{NA}$ & NA/NA & NA/NA & $\leq 0.12$ & $\leq 0.12$ \\
\hline & Ceftibuten & $\mathrm{NA} / \mathrm{NA}$ & $\mathrm{NA} / \mathrm{NA}$ & $\mathrm{NA} / \mathrm{NA}$ & 0.5 & 0.5 \\
\hline & Cefixime & NA/NA & NA/NA & NA/NA & $\leq 0.06$ & 0.12 \\
\hline & Cefpodoxime & NA/NA & NA/NA & NA/NA & $\leq 0.03$ & $\leq 0.03$ \\
\hline & Cefuroxime & $\mathrm{NA} / \mathrm{NA}$ & $\mathrm{NA} / \mathrm{NA}$ & $\mathrm{NA} / \mathrm{NA}$ & $\leq 0.03$ & $\leq 0.03$ \\
\hline & Cefaclor & NA/NA & NA/NA & NA/NA & 0.12 & 0.25 \\
\hline
\end{tabular}


Table 2 continued

\begin{tabular}{|c|c|c|c|c|c|c|}
\hline \multirow[t]{2}{*}{ Organism } & \multirow[t]{2}{*}{ Country $(n) /$ drug } & \multicolumn{3}{|c|}{ CLSI/EUCAST } & \multirow[t]{2}{*}{$\mathrm{MIC}_{50}$} & \multirow[t]{2}{*}{$\mathrm{MIC}_{90}$} \\
\hline & & $\% S$ & $\%$ I & $\% \mathbf{R}$ & & \\
\hline & Azithromycin & $81.8 / 0.0$ & $0.0 / 81.8$ & $18.2 / 18.2$ & $\leq 0.5$ & $>4$ \\
\hline & Clarithromycin & $81.8 / 81.8$ & $0.0 / 0.0$ & $18.2 / 18.2$ & $\leq 0.25$ & $>2$ \\
\hline & Ciprofloxacin & NA/NA & NA/NA & NA/NA & $\leq 0.12$ & 0.25 \\
\hline & Levofloxacin & $100 / 90.9$ & $0 / 9.1$ & $0.0 / 0.0$ & 1 & 1 \\
\hline & Moxifloxacin & NA/100 & $\mathrm{NA} / 0.0$ & $\mathrm{NA} / 0.0$ & $\leq 0.06$ & 0.12 \\
\hline & Doxycycline & $\mathrm{NA} / 81.8$ & $\mathrm{NA} / 0.0$ & $\mathrm{NA} / 18.2$ & $\leq 0.06$ & $>2$ \\
\hline & Trimethoprim/sulfamethoxazole & $\mathrm{NA} / 100$ & $\mathrm{NA} / 0.0$ & $\mathrm{NA} / 0.0$ & $\leq 0.12$ & $\leq 0.12$ \\
\hline \multirow[t]{15}{*}{ H. influenzae } & Argentina (10) & & & & & \\
\hline & Amoxicillin & NA/90.0 & $\mathrm{NA} / 0.0$ & NA/10.0 & 0.5 & 2 \\
\hline & Amoxicillin/clavulanic acid & $90.0 / 90.0$ & $0.0 / 0.0$ & $10.0 / 10.0$ & 1 & 2 \\
\hline & Ceftibuten & $100 / 100$ & $0.0 / 0.0$ & $0.0 / 0.0$ & 0.12 & 0.25 \\
\hline & Cefixime & $100 / 100$ & $0.0 / 0.0$ & $0.0 / 0.0$ & $\leq 0.015$ & 0.06 \\
\hline & Cefpodoxime & $100 / 100$ & $0.0 / 0.0$ & $0.0 / 0.0$ & 0.12 & 0.25 \\
\hline & Cefuroxime & $100 / 70.0$ & $0.0 / 20.0$ & $0.0 / 10.0$ & 0.5 & 2 \\
\hline & Cefaclor & $80.0 / 0.0$ & 20.0/0.0 & $0.0 / 100$ & 4 & 16 \\
\hline & Azithromycin & $100 / 0.0$ & $0.0 / 100$ & $0.0 / 0.0$ & 2 & 2 \\
\hline & Clarithromycin & $70.0 / 10.0$ & $30.0 / 90.0$ & $0.0 / 0.0$ & 8 & 16 \\
\hline & Ciprofloxacin & $100 / 100$ & $0.0 / 0.0$ & $0.0 / 0.0$ & 0.008 & 0.015 \\
\hline & Levofloxacin & $100 / 100$ & $0.0 / 0.0$ & $0.0 / 0.0$ & 0.03 & 0.03 \\
\hline & Moxifloxacin & $100 / 100$ & $0.0 / 0.0$ & $0.0 / 0.0$ & 0.015 & 0.015 \\
\hline & Doxycycline & NA/90.0 & $\mathrm{NA} / 10.0$ & $\mathrm{NA} / 0.0$ & $\leq 1$ & $\leq 1$ \\
\hline & Trimethoprim/sulfamethoxazole & $80.0 / 80.0$ & $0.0 / 0.0$ & $20.0 / 20.0$ & $\leq 0.25$ & $>4$ \\
\hline \multirow[t]{8}{*}{ H. influenzae } & Mexico (12) & & & & & \\
\hline & Amoxicillin & NA/58.3 & $\mathrm{NA} / 0$ & $\mathrm{NA} / 41.7$ & 1 & $>4$ \\
\hline & Amoxicillin/clavulanic acid & $100 / 83.3$ & $0.0 / 0.0$ & $0.0 / 16.7$ & 1 & 4 \\
\hline & Ceftibuten & $100 / 91.7$ & $0.0 / 0.0$ & $0.0 / 8.3$ & 0.12 & 0.25 \\
\hline & Cefixime & $100 / 91.7$ & $0.0 / 0.0$ & $0.0 / 8.3$ & 0.03 & 0.06 \\
\hline & Cefpodoxime & $100 / 83.3$ & $0.0 / 0.0$ & $0.0 / 16.7$ & 0.25 & 1 \\
\hline & Cefuroxime & $100 / 58.3$ & $0 / 41.8$ & $0.0 / 0.0$ & 1 & 2 \\
\hline & Cefaclor & $75.0 / 0.0$ & $16.7 / 0$ & $8.3 / 100$ & 4 & 16 \\
\hline
\end{tabular}


Table 2 continued

\begin{tabular}{|c|c|c|c|c|c|c|}
\hline \multirow[t]{2}{*}{ Organism } & \multirow[t]{2}{*}{ Country $(n) /$ drug } & \multicolumn{3}{|c|}{ CLSI/EUCAST } & \multirow[t]{2}{*}{$\mathrm{MIC}_{50}$} & \multirow[t]{2}{*}{$\mathrm{MIC}_{90}$} \\
\hline & & $\% S$ & $\%$ I & $\% \mathbf{R}$ & & \\
\hline & Azithromycin & $100 / 0.0$ & $0.0 / 100$ & $0.0 / 0.0$ & 2 & 4 \\
\hline & Clarithromycin & $66.7 / 0.0$ & $25 / 100$ & $8.3 / 0.0$ & 8 & 16 \\
\hline & Ciprofloxacin & $100 / 100$ & $0.0 / 0.0$ & $0.0 / 0.0$ & 0.008 & 0.015 \\
\hline & Levofloxacin & $100 / 100$ & $0.0 / 0.0$ & $0.0 / 0.0$ & 0.03 & 0.03 \\
\hline & Moxifloxacin & $100 / 100$ & $0.0 / 0.0$ & $0.0 / 0.0$ & 0.015 & 0.015 \\
\hline & Doxycycline & $0.0 / 100$ & $0.0 / 0.0$ & $0.0 / 0.0$ & $\leq 1$ & $\leq 1$ \\
\hline & Trimethoprim/sulfamethoxazole & $0.0 / 0.0$ & $0.0 / 0.0$ & $100 / 100$ & $>4$ & $>4$ \\
\hline \multirow[t]{15}{*}{ H. influenzae } & Russia (36) & & & & & \\
\hline & Amoxicillin & NA/94.4 & $\mathrm{NA} / 0.0$ & $\mathrm{NA} / 5.6$ & 0.25 & 0.5 \\
\hline & Amoxicillin/clavulanic acid & $100 / 100$ & $0.0 / 0.0$ & $0.0 / 0.0$ & 0.25 & 0.5 \\
\hline & Ceftibuten & $100 / 100$ & $0.0 / 0.0$ & $0.0 / 0.0$ & $\leq 0.03$ & 0.06 \\
\hline & Cefixime & $100 / 100$ & $0.0 / 0.0$ & $0.0 / 0.0$ & $\leq 0.015$ & 0.03 \\
\hline & Cefpodoxime & $100 / 100$ & $0.0 / 0.0$ & $0.0 / 0.0$ & $\leq 0.03$ & 0.06 \\
\hline & Cefuroxime & $100 / 100$ & $0.0 / 0.0$ & $0.0 / 0.0$ & 0.25 & 0.5 \\
\hline & Cefaclor & $100 / 0.0$ & $0.0 / 0.0$ & $0.0 / 100$ & 2 & 4 \\
\hline & Azithromycin & $100 / 0.0$ & $0 / 100$ & $0.0 / 0.0$ & 2 & 2 \\
\hline & Clarithromycin & $77.8 / 2.8$ & $19.4 / 97.2$ & $2.8 / 0.0$ & 8 & 16 \\
\hline & Ciprofloxacin & $100 / 100$ & $0.0 / 0.0$ & $0.0 / 0.0$ & 0.008 & 0.015 \\
\hline & Levofloxacin & $100 / 100$ & $0.0 / 0.0$ & $0.0 / 0.0$ & 0.03 & 0.03 \\
\hline & Moxifloxacin & $100 / 100$ & $0.0 / 0.0$ & $0.0 / 0.0$ & $\leq 0.008$ & 0.015 \\
\hline & Doxycycline & $0.0 / 100$ & $0.0 / 0.0$ & $0.0 / 0.0$ & $\leq 1$ & $\leq 1$ \\
\hline & Trimethoprim/sulfamethoxazole & $63.9 / 63.9$ & $2.8 / 0.0$ & $33.3 / 36.1$ & $\leq 0.25$ & $>4$ \\
\hline \multirow[t]{9}{*}{ H. influenzae } & Philippines (19) & & & & & \\
\hline & Amoxicillin & NA/89.5 & NA/0.0 & $\mathrm{NA} / 10.5$ & 0.5 & 4 \\
\hline & Amoxicillin/clavulanic acid & $100 / 100$ & $0.0 / 0.0$ & $0.0 / 0.0$ & 0.5 & 0.5 \\
\hline & Ceftibuten & $100 / 100$ & $0.0 / 0.0$ & $0.0 / 0.0$ & $\leq 0.03$ & 0.06 \\
\hline & Cefixime & $100 / 100$ & $0.0 / 0.0$ & $0.0 / 0.0$ & $\leq 0.015$ & 0.03 \\
\hline & Cefpodoxime & $100 / 100$ & $0.0 / 0.0$ & $0.0 / 0.0$ & 0.06 & 0.12 \\
\hline & Cefuroxime & $100 / 84.2$ & $0.0 / 15.8$ & $0.0 / 0.0$ & 0.5 & 2 \\
\hline & Cefaclor & $100 / 0.0$ & $0.0 / 0.0$ & $0.0 / 100$ & 4 & 8 \\
\hline & Azithromycin & $100 / 0.0$ & $0.0 / 100$ & $0.0 / 0.0$ & 2 & 4 \\
\hline
\end{tabular}


Table 2 continued

\begin{tabular}{|c|c|c|c|c|c|c|}
\hline \multirow[t]{2}{*}{ Organism } & \multirow[t]{2}{*}{ Country $(n) /$ drug } & \multicolumn{3}{|c|}{ CLSI/EUCAST } & \multirow[t]{2}{*}{$\mathrm{MIC}_{50}$} & \multirow[t]{2}{*}{$\mathrm{MIC}_{90}$} \\
\hline & & $\% S$ & $\%$ I & $\% \mathrm{R}$ & & \\
\hline & Clarithromycin & $31.6 / 0.0$ & $68.4 / 100$ & $0.0 / 0.0$ & 16 & 16 \\
\hline & Ciprofloxacin & $100 / 100$ & $0.0 / 0.0$ & $0.0 / 0.0$ & 0.008 & 0.015 \\
\hline & Levofloxacin & $100 / 100$ & $0.0 / 0.0$ & $0.0 / 0.0$ & 0.03 & 0.03 \\
\hline & Moxifloxacin & $100 / 100$ & $0.0 / 0.0$ & $0.0 / 0.0$ & 0.015 & 0.03 \\
\hline & Doxycycline & NA/100 & $\mathrm{NA} / 0.0$ & $\mathrm{NA} / 0.0$ & $\leq 1$ & $\leq 1$ \\
\hline & Trimethoprim/sulfamethoxazole & $31.6 / 31.6$ & $5.3 / 0.0$ & $63.2 / 68.4$ & $>4$ & $>4$ \\
\hline \multirow[t]{15}{*}{ M. catarrbalis } & Argentina (11) & & & & & \\
\hline & Amoxicillin & NA/NA & NA/NA & NA/NA & $\leq 0.12$ & $\leq 0.12$ \\
\hline & Amoxicillin/clavulanic acid & $100 / 100$ & $0.0 / 0.0$ & $\mathrm{NA} / 0.0$ & $\leq 0.12$ & $\leq 0.12$ \\
\hline & Ceftibuten & $100 / 100$ & $0.0 / 0.0$ & $\mathrm{NA} / 0.0$ & $\leq 0.03$ & $\leq 0.03$ \\
\hline & Cefixime & NA/NA & NA/NA & NA/NA & $\leq 0.03$ & $\leq 0.03$ \\
\hline & Cefpodoxime & NA/NA & NA/NA & NA/NA & 0.5 & 0.5 \\
\hline & Cefuroxime & $100 / 100$ & $0.0 / 0.0$ & NA/0.0 & $\leq 0.12$ & 1 \\
\hline & Cefaclor & $\mathrm{NA} / 100$ & $\mathrm{NA} / 0.0$ & $\mathrm{NA} / 0.0$ & $\leq 0.06$ & 0.12 \\
\hline & Azithromycin & $100 / 100$ & $0.0 / 0.0$ & $0.0 / 0.0$ & $\leq 0.5$ & $\leq 0.5$ \\
\hline & Clarithromycin & NA/100 & $\mathrm{NA} / 0.0$ & $\mathrm{NA} / 0.0$ & $\leq 0.06$ & $\leq 0.06$ \\
\hline & Ciprofloxacin & $63.6 / 9.1$ & $36.4 / 9.1$ & $0.0 / 81.8$ & $\leq 0.25$ & $\leq 0.25$ \\
\hline & Levofloxacin & $\mathrm{NA} / 100$ & $\mathrm{NA} / 0.0$ & $\mathrm{NA} / 0.0$ & $\leq 0.06$ & 0.25 \\
\hline & Moxifloxacin & $100 / 100$ & $0.0 / 0.0$ & NA/0.0 & $\leq 0.12$ & $\leq 0.12$ \\
\hline & Doxycycline & $100 / 100$ & $0.0 / 0.0$ & $0.0 / 0.0$ & 1 & 4 \\
\hline & Trimethoprim/sulfamethoxazole & NA/NA & NA/NA & NA/NA & $\leq 0.12$ & $\leq 0.12$ \\
\hline
\end{tabular}

CLSI Clinical Laboratory Standards Institute, EUCAST European Committee for Antimicrobial Susceptibility Testing, $I$ intermediate, $M I C$ minimal inhibitory concentrations, $R$ resistant, $S$ susceptible, $C A$ - $U R T I$ community-acquired upper respiratory tract infection, $N A$ no interpretive breakpoints available

observed in the Philippines and fluoroquinolone non-susceptible isolates were observed in all three countries that submitted $S$. pyogenes.

Among the 47 Enterobacteriaceae collected from CA-URTI using $\mathrm{MIC}_{50}$ values, ceftibuten, cefixime, and fluoroquinolones had similar potency $(0.03-0.12 \mu \mathrm{g} / \mathrm{mL})$.

\section{DISCUSSION}

This study determined the activity of oral antimicrobial agents from countries with limited information on the susceptibility patterns for pathogens which cause CA-UTI and CA-URTI. Oral cephalosporins tested in this study had activity against the majority of 
Enterobacteriaceae. Susceptibility rates were dependent on the breakpoints that were applied. Ceftibuten provided the highest and similar susceptibility rates using either CLSI or EUCAST breakpoint criteria against CA-UTI pathogens when compared to the oral cephalosporins tested. Amoxicillin-clavulanic acid susceptibility was much lower when using CLSI breakpoints compared to that observed using EUCAST uncomplicated UTI breakpoint criteria for this agent. Country variability in susceptibility was also observed, even within the same region with a $20 \%$ difference observed for fluoroquinolone susceptibility among the Latin American countries examined.

Sites which routinely do not collect specimens for culture from outpatients with CA-URTI limited the number of pathogens collected for this indication and not all countries provided a representative sample for this investigation. Also the number of countries in the three regions studied would not represent the entire region. Broad spectrum antimicrobial agents including all of those reported in this study should be used selectively. Cephalosporins and fluoroquinolones may not always be the first choice of therapy; due to the antibiotic pressure, these and other classes have on increasing ESBL rates. However, depending on the source of the infection and the pharmacokinetic and pharmacodynamics of the drug class, the loss of activity among other commonly used oral agents warrants the need to continue to monitor the viability of oral cephalosporins and other drugs used for treating outpatient infections.

It is important that local epidemiology efforts continue to determine the rate of emerging or epidemic clones and the resistance rates among several class agents which vary based on the susceptibility breakpoints applied [19-21]. It is equally important to follow the variation of prescribing practices and to reduce antibiotic consumption in the ambulatory setting as this affects antimicrobial resistance in the hospital setting [22, 23].

\section{CONCLUSION}

Information related to the local and regional susceptibility patterns of pathogens causing CA-UTI and CA-URTI is essential for physicians treating patients with these very common infections and that was the intent of this study. Continued surveillance of the pathogens causing CA-UTI and CA-URTI is necessary in this era of increasing antimicrobial resistance. Antimicrobial stewardship should remain a high priority across all countries to promote the best treatment practices to diminish the problem of antimicrobial resistance.

\section{ACKNOWLEDGMENTS}

Sponsorship and article processing charges for this study were funded by Merck \& Co. Inc., Kenilworth, New Jersey, USA as part of a sponsored surveillance program approved by the sponsor. We appreciate the effort of the country investigators and all laboratory staff who contributed towards isolate testing and the contribution towards our results. Flavia Rossi (University Sao Paulo) provided significant review of the protocol design. Investigators of this study included: Sara Celia Kaufman (Hospital Juan A. Fernandez), Jorgelina Smayvesky (CEMIC), Evelina N. Lagamayo (St. Luke's Medical Center), Federico Naranjo Briceno (Hospital Dr. Miguel Perez Carreno), Manuel Guzman Blanco (Centro medico de Caracas), Gustavo Barriga Angulo (Hospital de 
Infectologia). Thomas Lynch provided all the logistical support involved in providing protocols and support to the investigators. All named authors meet the International Committee of Medical Journal Editors (ICMJE) criteria for authorship for this manuscript, take responsibility for the integrity of the work as a whole, and have given final approval for the version to be published.

Disclosures. D. J. Biedenbach and R. E. Badal employees of International Health Management Associates, Inc., Schaumburg and claim no conflict of interest. R. S. Kozlov and A. D. Roman contributed to the study design, collection and analysis of data and receive honoraria for this study. M. Y. Huang, M. Motyl, P. K. Singhal and S. Marcella are employees of Merck \& Co. Inc., Kenilworth, New Jersey, USA and have financial interests.

Compliance with Ethics Guidelines. This article does not contain any new studies with human or animal subjects performed by any of the authors.

Open Access. This article is distributed under the terms of the Creative Commons Attribution-NonCommercial 4.0 International License (http://creativecommons.org/licenses/ by-nc/4.0/), which permits any noncommercial use, distribution, and reproduction in any medium, provided you give appropriate credit to the original author(s) and the source, provide a link to the Creative Commons license, and indicate if changes were made.

\section{REFERENCES}

1. Bantar C, Curcio D, Jasovich A, et al. Updated acute community-acquired pneumonia in adults: Guidelines for initial antimicrobial therapy based on local evidence from the South American Working Group (ConsenSur II). Rev Chilena Infectol. 2010;27(Suppl 1):S9-38.

2. Bours PH, Polak R, Hoepelman AI, Delgado E, Jarquin A, Matute AJ. Increasing resistance in community-acquired urinary tract infections in Latin America, five years after the implementation of national therapeutic guidelines. Int J Infect Dis. 2010;14:770-4.

3. Christiaens T, De Backer D, Burgers J, Baerheim A. Guidelines, evidence, and cultural factors: comparison of four European guidelines on uncomplicated cystitis. Scan J Prim Health Care. 2014;22:141-5.

4. Llor C, Rabanaque G, Lopez A, Cots JM. The adherence of GPs to guidelines for the diagnosis and treatment of lower urinary tract infections in women is poor. Fam Pract. 2011;28:294-9.

5. Rogue F, Soares S, Breitenfeld L, Figueiras A, Herdeiro MT. Influence of community parmacists' attitudes on antibiotic dispensing behaviour: a cross-sectional study in Portugal. Clin Ther. 2014;37:168-77.

6. Sanchez GV, Robers RM, Albert AP, Johnson DD, Hicks LA. Effects of knowledge, attitudes and practices of primary care providers on antibiotic selection, United States. Emerg Infect Dis. 2014;20:2041-7.

7. Stamm WE, Norrby SR. Urinary tract infections: disease panorama and challenges. J Infect Dis. 2001;183(Suppl 1):S1-4.

8. Ahmed NH, Hussain T, Biswal I. Comparison of etiological agents and resistance patterns of the pathogens causing community acquired and hospital acquired urinary tract infections. J Glob Infect Dis. 2014;6:135-6.

9. De Backer D, Christiaens T, Heytens S, De Sutter A, Stobberingh EE, Verschragen G. Evolution of bacterial susceptibility pattern of Escherichia coli in uncomplicated urinary tract infections in a country with high antibiotic consumption: a comparison of two surveys with a 10 year interval. $\mathrm{J}$ Antimicrob Chemother. 2008;62:364-8.

10. Abreu AG, Marques SG, Monteiro-Neto V, Goncalves AG. $\quad$ Extended-spectrum $\beta$-lactamase-producing enterobacteriaceae in community-acquired urinary tract infections in Sao Luis, Brazil. Braz J Microbiol. 2013;44:469-71.

11. Schaeffer EM. Extended-spectrum $\beta$-lactamase-producing gram-negative pathogens in community-acquired urinary tract infections: 
an increasing challenge for antimicrobial therapy. J Urol. 2012;188:831-2.

12. Kimura K, Nagano N, Nagano $\mathrm{Y}$, et al. High frequency of fluoroquinolone- and macrolide-resistant streptococci among clinically isolated group B streptococci with reduced penicillin susceptibility. J Antimicrob Chemother. 2013;68:539-42.

13. Golden AR, Rosenthal $\mathrm{M}$, Fultz $\mathrm{B}$, et al. Characterization of MDR and XDR Streptococcus pneumoniae in Canada, 2007-13. J Antimicrob Chemother. 2015;70:2199-202.

14. Lo DS, Shieh HH, Ragazzi SL, Koch VH, Martinez $\mathrm{MB}$, Gilio AE. Community-acquired urinary tract infection: age and gender-dependent etiology. J Bras Nefrol. 2013;35:93-8.

15. Rocha JL, Tuon FF, Johnson JR. Sex, drugs, bugs, and age: rational selection of empirical therapy for outpatient urinary tract infection in an era of extensive antimicrobial resistance. Braz J Infect Dis. $2012 ; 16: 115-21$.

16. Clinical Laboratory Standards Institute. 2014. Performance Standards for Antimicrobial Susceptibility Testing; Twenty-fourth Informational Supplement. CLSI document M100-S24. Clinical and Laboratory Standards Institute (CLSI), Wayne, PA 19087-1898 USA.

17. The European Committee on Antimicrobial Susceptibility Testing (EUCAST). Breakpoint tables for interpretation of MICs and zone diameters. Version 4.0, January 1, 2014. http:// www.eucast.org/clinical_breakpoints/. Accessed 28 Jan 2014
18. Clinical Laboratory Standards Institute. 2012. Methods for dilution antimicrobial susceptibility tests for bacteria that grow aerobically; Approved Standards-Ninth Edition. CLSI document M07-A9. Wayne, PA 19087-1898 USA.

19. Linhares I, Raposo $T$, Rodriques A, Almeida A. Frequency and antimicrobial resistance patterns of bacteria implicated in community urinary tract infections: a ten-year surveillance study (2000-2009). BMC Infect Dis. 2013;13:19.

20. Lopez-Cerero L, Navarro MD, Bellido M, et al. Escherichia coli belonging to the worldwide emerging epidemic clonal group O25b/ST131: risk factors and clinical implications. J Antimicrob Chemother. 2014;69:809-14.

21. Sorlozano A, Jimenez-Pacheco A, de Dios Luna Del Castillo J, et al. Evolution of the resistance to antibiotics of bacteria involved in urinary tract infections: A 7-year surveillance study. Am J Infect Control. 2014;42:1033-8.

22. Cordoba G, Siersma V, Lopez-Valcarcel B, et al. Prescribing style and variation in antibiotic prescriptions for sore throat: cross-sectional study across six countries. BMC Fam Pract. 2015;16:7.

23. Hürlimann D, Limacher A, Schabel M, Zanetti G, Berger C, Mühlemann K, Kronenberg A, Swiss Sentinel Working Group. Improvement of antibiotic prescription in outpatient care: a cluster-randomized intervention study using a sentinel surveillance network of physicians. J Antimicrob Chemother. 2015;70:602-8. 